Probability and Mathematical Physics: A Volume in Honor of Stanislav Molchanov (edited by D. Dawson, V. Jaksic, and B. Vainberg), vol. 42 of CRM Proceedings and Lecture Notes. Centre de Recherches Mathématiques, Montreal, pp. 113-130.

\title{
ASYMPTOTICS OF THE POINCARÉ FUNCTIONS
}

\author{
GREGORY DERFEL, PETER J. GRABNER $\dagger$, AND FRITZ VOGL
}

Dedicated to Prof. Stanislav A. Molchanov on the occasion of his $65^{\text {th }}$ birthday

\begin{abstract}
The asymptotic behaviour of the solutions of of Poincaré's functional equation $f(\lambda z)=P(f(z))(\lambda \in \mathbb{C},|\lambda|>1)$ for $P$ a polynomial of degree $\geq 2$ is studied in different regions of the complex plane.
\end{abstract}

\section{INTRODUCTION}

In $1890 \mathrm{H}$. Poincaré [12] has studied the equation

$$
f(\lambda z)=R(f(z)), \quad z \in \mathbb{C},
$$

where $R(z)$ is a rational function and $\lambda \in \mathbb{C}$. He proved that, if $R(0)=0, R^{\prime}(0)=\lambda$ and $|\lambda|>1$, then there exists a meromorphic or entire solution of (1.1). After Poincaré, solutions of (1.1) are called the Poincaré functions admitting a multiplication theorem (cf. [16]). Later on, G. Valiron $[15,16]$ elaborated the case, where $R(z)=P(z)$ is a polynomial, i.e.

$$
f(\lambda z)=P(f(z)), \quad z \in \mathbb{C},
$$

and obtained conditions for the existence of an entire solution $f(z)$. Furthermore, he derived the following asymptotic formula for $M(r)=\max _{|z|=r}|f(z)|$ :

$$
\log M(r) \sim r^{\rho} Q\left(\frac{\log r}{\log |\lambda|}\right), \quad r \rightarrow \infty .
$$

Here $Q$ is a 1-periodic function bounded between two positive constants, $\rho=\frac{\log m}{\log |\lambda|}$ and $m=\operatorname{deg} P(z)$.

An interesting example of such equations, which stems from the description of Brownian motion on Sierpinski's gasket, has been studied in ([1], [7], and [8]). This is the functional equation

$$
f(5 z)=4 f^{2}(z)-3 f(z) .
$$

Date: February 14, 2008.

2000 Mathematics Subject Classification. Primary: 30D05; Secondary: 39B32, 30D15, 37F10.

$\uparrow$ This author is supported by the Austrian Science Foundation FWF, project S9605, part of the Austrian National Research Network "Analytic Combinatorics and Probabilistic Number Theory".

This research was completed during the second author's visit to the Ben Gurion University of the Negev with support by the Center of Advanced Studies. 
Similar equations arise also in the theory of branching processes (cf. [2], [9]).

Different aspects of the Poincaré functions are discussed in papers [3], [5], [6], and [13] closely related to the present one.

In our paper we derive further results of Valiron's type. Namely, in addition to (1.3) we find asymptotics of entire solutions $f(z)$ on various rays $\arg z=\vartheta$ of the complex plane. It turns out that this heavily depends on the arithmetic nature of $\lambda$.

For instance, the following statement (Theorem 4.3, below) is proved:

If $\arg \lambda=2 \pi \beta$ and $\beta$ is irrational, then $f(z)$ is unbounded along any ray $\arg z=\vartheta$. Moreover, if we denote $\varphi(z)=\log |f(z)|$ (where the main branch of logarithm is taken) then there exists a sequence $r_{n} \rightarrow \infty$, such that the limit

$$
\lim _{n \rightarrow \infty} \frac{\varphi\left(r_{n} e^{i \vartheta}\right)}{r_{n}^{\rho}}=L
$$

exists and $L>0$.

On the other hand, if $\beta$ is rational (and, in particular, if $\beta=0$, i.e. $\lambda$ is real) $f(z)$ may be bounded on some rays and even in whole sectors. Nevertheless, for rational $\beta$, the limit (1.5) still exists under some additional assumptions. Denote $\beta=t / s$ and suppose that $t, s$ are relatively prime. Put $q=\lambda^{s}$ (note that $1<q \in \mathbb{R}$ ). Here we have the following result (see Theorems 5.1 and 5.2 below):

Suppose that either $|\lambda|>m^{2}$ or $s>2 \rho$. Then $f(z)$ is unbounded on any ray, and one can find a geometric progression $r_{n}=q^{n} r_{0},\left(r_{0}>0\right)$, for which the limit (1.5) exists and $L>0$.

Further refinements are possible when $\lambda>1$ is real and $P(z)=p_{m} z^{m}+\ldots+p_{1} z+$ $p_{0}$ is a polynomial with real coefficients. Namely, we prove the the following statement (Theorem 6.1):

Assume that: a) $\lambda>m$; b) $p_{i} \geq 0$, for $i \geq 2$; c) All preimages of 0 under $P$, i.e $P^{-n}(\{0\})$ are real. Let $f(z)$ be an entire solution of $(1.2)$ such that $f(0)$ and $f^{\prime}(0)$ are real and $f(0) \geq 0$ and $f^{\prime}(0)>0$. Then $f(z)$ tends to infinity along any ray in the sector $0<|\vartheta|<\pi / 2$. Moreover,

$$
\varphi\left(r e^{i \vartheta}\right) \sim r^{\rho} Q_{\vartheta}\left(\frac{\log r}{\log \lambda}\right), \quad r \rightarrow \infty
$$

where $Q_{\vartheta}(z)$ is 1-periodic and bounded between two positive constants.

Condition c) plays an important role in the last statement. If $P(z)$ is quadratic polynomial (a case, arising in some applications) it is possible to give an exact criterion for reality of $P^{-n}(\{0\})$ (see Lemma 6.4 below): Let

$$
P(z)=a z(z-\omega), \quad 0 \neq \omega \in \mathbb{R}
$$

All preimages of 0 under $P$ are real, if and only if the following condition is fulfilled

$$
a|\omega| \geq \begin{cases}2 & \text { for } \omega>0 \\ 4 & \text { for } \omega<0\end{cases}
$$


The paper is organised as follows. In section 2 we discuss some elementary but important properties of the Poincaré equation (1.2) and its (formal) power series solutions. Section 3 is devoted to a study of the asymptotics of solutions for (1.2) along spirals (i.e. geometric progression of the form $z_{n}=\lambda^{n} z_{0}, \lambda \in \mathbb{C}$ ). As a consequence of these results, in Section 4 , we obtain asymptotics of solutions on different rays of the complex plane. We split the presentation into two different cases:

a) $\beta=\frac{1}{2 \pi} \arg \lambda$ is rational (Section 4.1)

b) $\beta$ is irrational (Section 4.2)

Sufficient conditions for unboundedness of solutions along all rays emanating from the origin are given in Section 5 .

Further refinements are possible, when $\lambda>1$ is real and $P(z)$ is a polynomial with real and "almost positive" coefficients - these are derived in Section 6. Also, we specialise some of our results for quadratic polynomials.

In the final Section 7 we present some results on the asymptotics of solutions $f(z)$ (rather than $|f(z)|)$ in some angular regions of the complex plane.

\section{Preliminary REMARKs, Simplifications of the EQUATION, FORMAL POWER SERIES SOLUTION}

Consider the equation

$$
f(\lambda z)=P[f(z)]
$$

where

$$
P(z)=p_{m} z^{m}+p_{m-1} z^{m-1}+\ldots+p_{0} .
$$

Throughout this paper we assume that $m \geq 2$ and $|\lambda|>1$.

2.1. Simplifications of the equation. The following Proposition 2.1 shows that, without loss of generality, we may always assume that $p_{m}=1$.

Proposition 2.1. Let $f(z)$ be a solution for (2.1). Then

$$
g(z)=p_{m}^{-\frac{1}{m-1}} f(z)
$$

is a solution of the equation

$$
g(\lambda z)=\widetilde{P}[g(z)]
$$

where

$$
\widetilde{P}(z)=z^{m}+\widetilde{p}_{m-1} z^{m-1}+\ldots+\widetilde{p}_{0},
$$

is a polynomial with the leading coefficient equal to 1.

(More precisely,

$$
\widetilde{p}_{k}=p_{k} c^{k-1} ; \text { for } k=0, \ldots m \text { with } c=p_{m}^{-\frac{1}{m-1}} \text {, }
$$

In particular, $\widetilde{p}_{0}=p_{0} c^{-1}=p_{0} p_{m}^{\frac{1}{m-1}}$.) 
Proof. Put $f(z)=c g(z)$ and insert this in (2.1).

Then we have

$$
c g(\lambda z)=p_{m} c^{m}[g(z)]^{m}+p_{m-1} c^{m-1}[g(z)]^{m-1}+\ldots+p_{0}
$$

or

$$
g(\lambda z)=p_{m-1} c^{m-1}[g(z)]^{m}+p_{m-1} c^{m-2}[g(z)]^{m-2}+\ldots+c^{-1} p_{0}
$$

Next, choose $c$ so that $p_{m} c^{m-1}=1$, i.e. $c=p_{m}^{-\frac{1}{m-1}}$. This completes the proof.

Example 1. By virtue of the formula $\cos 2 z=2 \cos ^{2} z-1$ the function $f(z)=\cos z$ satisfies the equation

$$
f(2 z)=2[f(z)]^{2}-1
$$

Here $p_{2}=2, c=p_{2}^{-1}=2^{-1}$ and therefore $\widetilde{p}_{2}=1, \widetilde{p}_{0}=-2$.

Therefrom, according to Proposition 2.1, the function $g(z)=2 \cos z=e^{i z}+e^{-i z}$ satisfies the equation

$$
g(2 z)=g(z)^{2}-2
$$

Proposition 2.2. Let $f(z)$ be a solution of (2.1). Then

$$
g(z)=f\left(z^{\gamma}\right), \text { for } \gamma>0
$$

is a solution of the equation

$$
g\left(\lambda^{1 / \gamma} z\right)=P[g(z)]
$$

Proof.

$$
g\left(\lambda^{1 / \gamma} z\right)=f\left[\left(\lambda^{1 / \gamma} z\right)^{\gamma}\right]=f\left(\lambda z^{\gamma}\right)=P\left[f\left(z^{\gamma}\right)\right]=P[g(z)]
$$

Example 2. As it was mentioned already the function $f(z)=\cos z$ satisfies equation

$$
f(2 z)=P[f(z)]
$$

where $P(z)=2 z^{2}-1$. Put $g(z)=\cos \sqrt{z}$, i.e. choose $\gamma=1 / 2$ in (2.6). Then, in accordance with Proposition 2.2, the (entire) function $g(z)=\cos \sqrt{z}$ satisfies the equation

$$
g(4 z)=\cos \sqrt{4 z}=\cos 2 \sqrt{z}=2(\cos \sqrt{z})^{2}-1=P[g(z)]
$$

H. Poincaré in $[11,12]$ and G. Valiron in [16] assumed that $P(0)=0$. There is no loss of generality in this assumption, as is shown in the following Proposition 2.3. The condition $P(0)=0$ can be always achieved by simple change of variables.

Proposition 2.3. Suppose that $f(z)$ satisfies equation (2.1) and initial condition

$$
f(0)=c_{0}
$$

(In accordance with Lemma 2.1 below, it is necessary that $c_{0}=P\left(c_{0}\right)$, i.e. $c_{0}$ is fixed point of $P(z)$.) Then the function $g(z)=f(z)-c_{0}$ satisfies the equation

$$
g(\lambda z)=\widetilde{P}[g(z)],
$$


where

$$
\widetilde{P}(z)=P\left(z+c_{0}\right)-c_{0}
$$

and the initial condition

$$
g(0)=0
$$

In addition,

$$
\widetilde{P}(0)=0
$$

i.e. 0 is a fixed point of $\widetilde{P}(z)$ (which corresponds to the fixed point $c_{0}$ of $P(z)$ ). Furthermore, if $c_{i}(i=0,1, \ldots m-1)$ are fixed points of $P(z)$, then $\widetilde{c}_{i}=c_{i}-c_{0}(i=0, \ldots, \ell)$ are fixed points of $\widetilde{P}(z)$ and $\widetilde{P}^{\prime}\left(\widetilde{c}_{i}\right)=P^{\prime}\left(c_{i}\right)$

Proof. Firstly

$$
g(\lambda z)=f(\lambda z)-c_{0}=P[f(z)]-c_{0}=P\left[g(z)+c_{0}\right]-c_{0} \equiv \widetilde{P}[g(z)]
$$

Also, clearly

$$
g(0)=f(0)-c_{0}=c_{0}-c_{0}=0 ; \quad \widetilde{P}(0)=P\left(c_{0}\right)-c_{0}=0
$$

Next, suppose that $c_{i}(i=0, \ldots, \ell)$ is a fixed point of $P(z)$. Then

$$
\widetilde{P}\left(c_{i}-c_{0}\right)=P\left[\left(c_{i}-c_{0}\right)+c_{0}\right]-c_{0}=P\left(c_{i}\right)-c_{0}=c_{i}-c_{0}
$$

i.e. $\widetilde{c}=c_{i}-c_{0}$ is a fixed point of $\widetilde{P}(z)$. Finally, $\widetilde{P}^{\prime}(z)=P^{\prime}\left(z+c_{0}\right)$ for any $z$ and, in particular, $\widetilde{P}^{\prime}\left(\widetilde{c}_{i}\right)=\widetilde{P}^{\prime}\left(c_{i}-c_{0}\right)=P^{\prime}\left(c_{i}\right)$.

2.2. Formal power series solutions of (2.1). . Let

$$
f(z)=\sum_{k=0}^{\infty} f_{k} z^{k}
$$

be a formal power series solution of (2.1). It is easily verified that $f(z)$ satisfies $(2.1)$ if and only if the coefficients $f_{n}$ satisfy the following recurrence

$$
\begin{aligned}
P\left(f_{0}\right) & =f_{0} \\
(\lambda-\alpha) f_{1} & =0, \text { with } \alpha:=P^{\prime}\left(f_{0}\right)=\sum_{k=1}^{m} k p_{k} f_{0}^{k-1} \\
\left(\lambda^{n}-\alpha\right) f_{n} & =\sum_{k=2}^{m} p_{k} \sum_{\substack{\ell_{1}+\ldots+\ell_{k}=n \\
\ell_{1}, \ldots, \ell_{k}<n}} f_{\ell_{1}} f_{\ell_{2}} \ldots f_{\ell_{K}}=: S_{n}\left(p_{2}, \ldots, p_{m}, f_{0}, f_{1}, \ldots f_{n-1}\right)
\end{aligned}
$$

Remark 2.1. We call a power series solution trivial, if it degenerates to the constant $f_{0}$. Note, that in view of (2.1), a nontrivial solution is non-polynomial.

The following Lemma immediately follows from (2.9)-(2.11).

Lemma 2.1. The following assertions hold:

(1) The only admissible values for $f_{0}$ (at most $m$ ) are the fixed points of $P(z)$. 
(2) A nontrivial formal power solution $f(z)$ exists if and only if there is an $n_{0} \in \mathbb{N}$ such that

$$
\lambda^{n_{0}}=\alpha=P^{\prime}\left(f_{0}\right)
$$

(3) If $n_{0} \geq 2$ and $f(z)$ is a nontrivial solution, then

$$
f_{k n_{0}+1}=f_{k n_{0}+2}=\ldots=f_{(k+1) n_{0}-1}=0 \text { for } k=0,1, \ldots
$$

and there are infinitely many $f_{i}$, such that $f_{i} \neq 0$.

(4) $S_{n}\left(p_{2}, \ldots, p_{m}, f_{0}, f_{1}, \ldots f_{n-1}\right)$ is a polynomial of its arguments with positive coefficients.

Remark 2.2. Let $f(z)$ be a nontrivial formal power solution of (2.1), which satisfies (2.12) with $n_{0} \geq 2$. Then, from Lemma 2.1-(3) it follows that:

(1) $f(z)$ is even, if $n_{0}$ is even

(2) the function

$$
g(z)=f\left(\sqrt[n_{0}]{z}\right)
$$

can be developed into a formal power series and satisfies the equation

$$
g\left(\lambda^{n_{0}} z\right)=P[g(z)]
$$

in a line with Proposition 2.2. In other words, $g(z)$ is formal power series solution of equation

$$
g(\mu z)=P[g(z)]
$$

where

$$
\mu:=\lambda^{n_{0}}=\alpha=P^{\prime}\left(g_{0}\right)=P^{\prime}\left(f_{0}\right)
$$

i.e. $g(z)$ satisfies $(2.12)$, with $n_{0}=1$

2.3. Entire solution versus formal solution. We conclude this section with the following classical theorem of Valiron.

Theorem 2.4 (Valiron [16]). Any formal power series solution $f(z)$ of $(2.1)$ is an entire function of order

$$
\rho=\frac{\log m}{\log |\lambda|}
$$

Furthermore, denote $M(r)=\max _{|z| \leq r}|f(z)|$. Then

$$
\log M(r) \sim r^{\rho} Q\left(\frac{\log r}{\log |\lambda|}\right), \quad r \rightarrow \infty,
$$

where $Q$ is a 1-periodic function bounded between two positive constants. 


\section{Asymptotics of SOlUtions AlONG SPIRALS}

As it was mentioned already in the introduction the main objective of this paper is to derive asymptotics for the solutions of (2.1) along different rays $\arg z=\vartheta$ of the complex plane $\mathbb{C}$.

Latter asymptotics can be derived from the asymptotics of $f(z)$ along spirals (geometric progressions) of the form $z_{n}=\lambda^{n} z_{0}$, where $z_{0}=r_{0} e^{i \vartheta_{0}} \in \mathbb{C}$ will be specified below. Corresponding results are given in Theorem 3.1, below.

Consider the Poincaré equation

$$
f(\lambda z)=P[f(z)], \quad|\lambda|>1
$$

where (as indicated in Section 2.1, we can always assume that $P$ is normalised)

$$
P(z)=z^{m}+p_{m-1} z^{m-1}+\ldots+p_{1} z+p_{0}, \quad m \geq 2 .
$$

Denote

$$
K=\max \left\{\left|p_{0}\right|, \ldots,\left|p_{m-1}\right|\right\} .
$$

Suppose that $z_{0}=r_{0} e^{i \vartheta_{0}} \in \mathbb{C}$ is such a point, that

$$
\left|f\left(z_{0}\right)\right|>\max \{e, 2 m K\} .
$$

Denote

$$
\varphi(z)=\log |f(z)| ; \quad z_{n}=\lambda^{n} z_{0} ; \quad \rho=\frac{\log m}{\log |\lambda|}
$$

(It follows from $(3.4)$ that $\varphi\left(z_{0}\right)>1$.)

Theorem 3.1. Suppose that $f(z)$ is a solution of (3.1) and (3.4) is satisfied. Then the limit along the spiral $z_{n}=\lambda^{n} z_{0}$

$$
\lim _{n \rightarrow \infty} \frac{\varphi\left(z_{n}\right)}{\left|z_{n}\right|^{\rho}}=L\left(z_{0}\right)
$$

exists and

$$
\frac{\varphi\left(z_{0}\right)-\frac{3 K m}{2\left|f\left(z_{0}\right)\right|}}{\left|z_{0}\right|^{\rho}}<L\left(z_{0}\right)<\frac{\varphi\left(z_{0}\right)+\frac{3 K m}{2\left|f\left(z_{0}\right)\right|}}{\left|z_{0}\right|^{\rho}} .
$$

In particular, in view of $(3.4), L\left(z_{0}\right)$ is bounded between two positive constants:

$$
\frac{\varphi\left(z_{0}\right)-\frac{3}{4}}{\left|z_{0}\right|^{\rho}}<L<\frac{\varphi\left(z_{0}\right)+\frac{3}{4}}{\left|z_{0}\right|^{\rho}}
$$

Furthermore, if $f(z)$ is an entire solution of (3.1), then $L$ is a continuous function on the domain $\{z \in \mathbb{C}|| f(z) \mid>\max (e, 2 m K)\}$.

Remark 3.1. Throughout this paper we deal mainly with the asymptotics of entire solutions of (3.1). However, some statements of the present section and Section 4 are valid for arbitrary solutions of (3.1). In particular, for validity of (3.6) and (3.7) no assumptions on the smoothness of $f(z)$ are needed.

The proof is similar to the one of [4, Lemma 1]. It may be divided into two main steps. We shall state these two steps as Lemmas 3.1 and 3.2 below. 
Lemma 3.1. Suppose that (3.4) is fulfilled. Then

$$
\log f\left(\lambda z_{0}\right)=m \log f\left(z_{0}\right)+r\left(z_{0}\right),
$$

where

and

$$
r(z)=\log \left(1+\frac{p_{m-1}}{f(z)}+\cdots+\frac{p_{0}}{f(z)^{m}}\right)
$$

$$
\left|r\left(z_{0}\right)\right| \leq \frac{3 m K}{2\left|f\left(z_{0}\right)\right|}
$$

Denote $\Re r\left(z_{0}\right)=r_{1}\left(z_{0}\right)$ and equate real parts in (3.9).

As an immediate consequence of of Lemma 3.1 we obtain

Corollary 3.1. If (3.4) is satisfied, then

$$
\log \left|f\left(\lambda z_{0}\right)\right|=m \log \left|f\left(z_{0}\right)\right|+r_{1}\left(z_{0}\right)
$$

or

where

$$
\varphi\left(\lambda z_{0}\right)=m \varphi\left(z_{0}\right)+r_{1}\left(z_{0}\right)
$$

$$
\left|r_{1}\left(z_{0}\right)\right| \leq \frac{3 m K}{2\left|f\left(z_{0}\right)\right|}
$$

Proof of Lemma 3.1. Let us write $P(z)$ in the form

$$
P(z)=z^{m}\left(1+\frac{p_{m}}{z}+\cdots+\frac{p_{0}}{z^{m}}\right)=: z^{m}(1+R(z)) .
$$

Then we have for $|z| \geq 1$

From (3.14) it follows that

$$
|R(z)| \leq \frac{m K}{|z|}
$$

$$
\log P(z)=m \log z+\tilde{r}(z),
$$

where $\tilde{r}(z)=\log (1+R(z))$. Moreover, if (3.4) is satisfied, then

$$
\left|\tilde{r}\left(z_{0}\right)\right| \leq \frac{3}{2}\left|R\left(z_{0}\right)\right| \leq \frac{3}{2} \frac{m K}{\left|z_{0}\right|}
$$

(in view of the inequality $|\log (1+z)| \leq \frac{3}{2}|z|$, which is valid for $|z| \leq \frac{1}{2}$ ).

Next, combining (3.1) and (3.15), we obtain (3.9), where $r\left(z_{0}\right)=\tilde{r}\left(f\left(z_{0}\right)\right)$. Furthermore, it follows from (3.16) that $r\left(z_{0}\right)$ satisfies (3.10).

Remark 3.2. From the proof of Lemma 3.1 it follows that $r(z)$ is an analytic function and $r_{1}(z)$ is continuous in the domain $\{z \in \mathbb{C}|| f(z) \mid>\max (e, 2 m K)\}$, if $f(z)$ is an entire solution of (3.1).

Now, from Corollary 3.1 we derive the two simple additional Corollaries 3.2 and 3.3.

Corollary 3.2. The sequence $\varphi\left(\lambda^{n} z_{0}\right)$ is monotonically increasing and tends to infinity, as $n \rightarrow \infty$. Moreover $\varphi\left(\lambda^{n} z_{0}\right)>(5 / 4)^{n} \varphi\left(z_{0}\right)$. 
Proof. It follows from (3.4) that

$$
\varphi\left(z_{0}\right)>1 \text { and }\left|r_{1}\left(z_{0}\right)\right|<\frac{3}{4} .
$$

Combining (3.12), (3.13), and (3.17) we obtain

$$
\begin{aligned}
\varphi\left(\lambda z_{0}\right)=m\left(\varphi\left(z_{0}\right)+\frac{r_{1}\left(z_{0}\right)}{m}\right) & \geq m\left(\varphi\left(z_{0}\right)-\frac{3}{4 m}\right) \\
& \geq m\left(\varphi\left(z_{0}\right)-\frac{3}{4 m} \varphi\left(z_{0}\right)\right)=\frac{4 m-3}{4} \varphi\left(z_{0}\right) \geq \frac{5}{4} \varphi\left(z_{0}\right) .
\end{aligned}
$$

This implies monotonicity as well as the lower bound for $\varphi\left(\lambda^{n} z_{0}\right)$.

Combining Corollary 3.2 with the estimate (3.13) yields

Corollary 3.3. For $n=0,1, \ldots$ we have

$$
\left|r_{1}\left(\lambda^{n} z_{0}\right)\right|<\frac{3}{2} \frac{m K}{\left|f\left(z_{0}\right)\right|}=\frac{3}{2} m K e^{-\varphi\left(z_{0}\right)} .
$$

Lemma 3.2. Suppose that (3.4) is satisfied. Then the limit

$$
\lim _{n \rightarrow \infty} \frac{\varphi\left(\lambda^{n} z_{0}\right)}{m^{n}}=L_{1}\left(z_{0}\right)
$$

exists and

$$
\left|L_{1}\left(z_{0}\right)-\varphi\left(z_{0}\right)\right| \leq \frac{3 m K}{2\left|f\left(z_{0}\right)\right|}
$$

Furthermore, if $f$ is an entire solution, then $L$ is a continuous function on some neighbourhood of $z_{0}$.

Proof. From (3.12) we get by iteration

$$
\frac{\varphi\left(\lambda^{n} z_{0}\right)}{m^{n}}=\varphi\left(z_{0}\right)+\frac{1}{m} r_{1}\left(z_{0}\right)+\cdots+\frac{1}{m^{n}} r_{1}\left(\lambda^{n-1} z_{0}\right) .
$$

Denote

$$
S\left(z_{0}\right)=\sum_{k=1}^{\infty} \frac{r_{1}\left(\lambda^{k-1} z_{0}\right)}{m^{k}},
$$

where the series converges uniformly by (3.19) and

$$
\left|S\left(z_{0}\right)\right| \leq \frac{3 m K}{2\left|f\left(z_{0}\right)\right|} .
$$

Therefore the limit (3.20) exists and equals $\varphi\left(z_{0}\right)+S\left(z_{0}\right)$. The inequality (3.21) follows from (3.24). Continuity of $L_{1}$ is a consequence of uniform convergence of the series (3.23) and the continuity of $\varphi$ and $r_{1}$.

Now Theorem 3.1 is an immediate consequence of Lemma 3.1. 
Proof of Theorem 3.1. Since $z_{n}=\lambda^{n} z_{0}$ and $\rho=\frac{\log m}{\log |\lambda|}$ then $|\lambda|^{n}=\left|\frac{z_{n}}{z_{0}}\right|$ and $m=|\lambda|^{\rho}$. Therefore,

Inserting this into (3.20) we get

$$
m^{n}=\left(|\lambda|^{\rho}\right)^{n}=\left|\frac{z_{n}}{z_{0}}\right|^{\rho}
$$

$$
\lim _{n \rightarrow \infty} \frac{\varphi\left(z_{n}\right)}{\left|z_{n}\right|^{\rho}}=\lim _{n \rightarrow \infty} \frac{\varphi\left(z_{n}\right)}{\left|z_{0}\right|^{\rho} m^{n}}=L_{1}\left(z_{0}\right)\left|z_{0}\right|^{-\rho}=L\left(z_{0}\right) .
$$

Combining this with (3.21) we obtain

$$
\left|L\left(z_{0}\right)-\frac{\varphi\left(z_{0}\right)}{\left|z_{0}\right|^{\rho}}\right| \leq \frac{3 m K}{2\left|f\left(z_{0}\right)\right|\left|z_{0}\right|^{\rho}},
$$

which gives (3.7).

As can be seen from the proof, it is possible to relax the conditions of Theorem 3.1 slightly and to restate it in the following modified form.

Theorem 3.2. Suppose that $f(z)$ satisfies (3.11) (or, what is the same, $\varphi(z)$ satisfies (3.12)). Suppose further that (3.13) is satisfied. Then the conclusion of Theorem 3.1 holds, i.e. $\varphi(z)$ satisfies (3.6) and (3.7).

Remark 3.3. One can see from the proof (see (3.22)-(3.24)) that not only (3.26) (or (3.7)) is true, but the slightly stronger statement

$$
\left|\frac{\varphi\left(z_{n}\right)}{\left|z_{n}\right|^{\rho}}-\frac{\varphi\left(z_{0}\right)}{\left|z_{0}\right|^{\rho}}\right| \leq \frac{3 m K}{2\left|f\left(z_{0}\right)\right|\left|z_{0}\right|^{\rho}} \text { for } n=1,2, \ldots
$$

holds.

\section{Asymptotics Along RAys}

As a consequence of Theorem 3.1 on the asymptotic behaviour of $f(z)$ along spirals we shall derive the asymptotics along rays $r e^{i \vartheta}$ in this section. It turns out that the latter heavily depends on the arithmetic nature of $\arg \lambda$.

Denote $\arg \lambda=2 \pi \beta$. Then it is natural to distinguish two cases: $\beta$ is rational and $\beta$ is irrational.

\subsection{Rational angle.}

Theorem 4.1. Suppose that the conditions of Theorem 3.1 (or Theorem 3.2) are satisfied. Suppose further that $\beta=\frac{t}{s}$ is rational (with s and $t$ relatively prime). Denote $q=\lambda^{s}$ (note that $q$ is a real number $>1$ ); $r_{n}=q^{n} r_{0}$ (and recall the notation $z_{0}=r_{0} e^{i \vartheta_{0}}$ ). Then the limit

$$
\lim _{n \rightarrow \infty} \frac{\varphi\left(r_{n} e^{i \vartheta}\right)}{r_{n}^{\rho}}=L\left(z_{0}\right)
$$

exists and satisfies (3.7). 
Remark 4.1. Similar asymptotics occur on the rays

$$
\arg z=\vartheta_{0}+\frac{2 k \pi}{s}, \quad k=0,1, \ldots, s-1 .
$$

Proof. If $f(z)$ satisfies (3.1) then (by iteration of (3.1)) it also satisfies

$$
f\left(\lambda^{s} z\right)=P^{(s)}(f(z)),
$$

where $P^{(s)}$ denotes the $s$-th iteration of $P$. By our assumptions $q=\lambda^{s}$ is real and $>1$. The assertion follows by an application of Theorem 3.1 to $P^{(s)}$.

If in addition to the assumptions of Theorem 4.1 it is known that $f(z) \rightarrow \infty$ as $z \rightarrow \infty$ along the ray $\arg z=\vartheta_{0}$, then one can obtain stronger results. Suppose that there exists an interval $I=\left[r_{0}, r_{0} q\right]$ such that condition (3.4) is satisfied for all $z \in e^{i \vartheta_{0}} I$, i.e.

$$
\forall z \in e^{i \vartheta_{0}} I:|f(z)|>\max \{e, 2 m K\} .
$$

Denote

$$
M_{0}=\max _{z \in e^{i \vartheta_{0} I}} \varphi(z) \text { and } m_{0}=\min _{z \in e^{i \vartheta_{0} I}} \varphi(z) .
$$

Then the following is an immediate consequence of Theorem 4.1.

Theorem 4.2. Suppose that the assumptions of Theorem 3.1 (or Theorem 3.2) are satisfied, $\beta=\frac{t}{s}$ is rational and (4.3) holds. Then

$$
\varphi\left(r e^{i \vartheta_{0}}\right) \sim r^{\rho} Q\left(\frac{\log r}{\log q}\right)
$$

where $Q$ is a continuous 1-periodic real function of a real variable bounded between two positive constants:

$$
\forall t \in \mathbb{R}: \frac{m_{0}-\frac{3}{4}}{\left(q r_{0}\right)^{\rho}} \leq Q(t) \leq \frac{M_{0}+\frac{3}{4}}{r_{0}^{\rho}}
$$

Remark 4.2. The theorem of Valiron [16] cited in Section 1 follows from Theorem 4.2. Proof. Suppose that $f(z)$ is an entire solution of (3.1). Denote

$$
M(r)=\max _{|z|=r}|f(z)| \text { and } \psi(r)=\log M(r) .
$$

Then arguments analogous to those which led us to (3.12) and (3.13) yield

$$
\psi(|\lambda| r)=m \psi(r)+R(r)
$$

with

$$
|R(r)|<\frac{3 m K}{2 M(r)}
$$

Thus the conditions of Theorem 3.2 are satisfied for the real scaling factor $|\lambda|$ in (4.6). Clearly, also $M(r)>\max \{e, 2 m K\}$ for $r$ large enough, which implies (4.3). From this we obtain

which implies Valiron's theorem.

$$
\log M(r) \sim r^{\rho} Q\left(\frac{\log r}{\log |\lambda|}\right)
$$


4.2. Irrational angle. In this case we cannot claim the existence of geometric progressions along a given ray, for which (4.1) holds. Instead we will prove that for any ray $r e^{i \vartheta}$ there exists a sequence of positive real numbers $\left(s_{n}\right)$ with $s_{n} \rightarrow \infty$ such that

$$
\lim _{n \rightarrow \infty} \frac{\varphi\left(s_{n} e^{i \vartheta}\right)}{s_{n}^{\rho}}=L>0
$$

exists. In particular, this implies that in the case of irrational $\beta$ solutions of the Poincaré equation are unbounded on any ray. As far as we know this phenomenon has not been mentioned in the literature before. An alternative proof of the latter statement based on the Phragmén-Lindelöf principle will be given in Section 5 .

Theorem 4.3. Suppose that the assumptions of Theorem 3.1 (or Theorem 3.2) are satisfied and $\beta=\frac{1}{2 \pi} \arg \lambda$ is irrational. Then on any ray $\arg z=\vartheta$ there exists a sequence $\left(s_{n} e^{i \vartheta}\right)$ with $s_{n} \rightarrow \infty$ such that the limit

$$
\lim _{n \rightarrow \infty} \frac{\varphi\left(s_{n} e^{i \vartheta}\right)}{s_{n}^{\rho}}=L\left(z_{0}\right)
$$

exists and satisfies (3.7).

Proof. Let $z_{0}$ satisfy (3.4). Then there exists an $\varepsilon>0$ such that $|f(z)|>\max (e, 2 m K)$ holds for all $z \in B\left(z_{0}, \varepsilon\right)$. By Lemma 3.2

$$
L_{1}(z)=\lim _{n \rightarrow \infty} \frac{\varphi\left(\lambda^{n} z\right)}{m^{n}}
$$

is a continuous function of $z$ on $B\left(z_{0}, \varepsilon\right)$ and convergence is uniform in $B\left(z_{0}, \varepsilon\right)$ as indicated in the proof of Lemma 3.2.

By the density of the set $\{n \arg \lambda \mid n \in \mathbb{N}\}$ on the unit circle there exists a sequence of integers $\left(n_{k}\right)_{k \in \mathbb{N}}$ such that

$$
\lim _{k \rightarrow \infty} \arg \lambda^{n_{k}}=\vartheta-\arg z_{0} \bmod 2 \pi .
$$

Let

$$
\zeta_{k}=\left|z_{0}\right| e^{i \vartheta}\left(\frac{\lambda}{|\lambda|}\right)^{-n_{k}}
$$

Then clearly $\lim _{k \rightarrow \infty} \zeta_{k}=z_{0}$ and we have

$$
\lim _{k \rightarrow \infty} \frac{\varphi\left(\lambda^{n_{k}} \zeta_{k}\right)}{m^{n_{k}}}=\lim _{k \rightarrow \infty} \frac{\varphi\left(\left|z_{0}\right| e^{i \vartheta}|\lambda|^{n_{k}}\right)}{m^{n_{k}}}=L_{1}\left(z_{0}\right)
$$

by uniform convergence. Denoting $s_{k}=\left|z_{0}\right||\lambda|^{n_{k}}$ we have then

$$
\lim _{k \rightarrow \infty} \frac{\varphi\left(s_{k} e^{i \vartheta}\right)}{s_{k}^{\rho}}=\lim _{k \rightarrow \infty} \frac{\varphi\left(s_{k} e^{i \vartheta}\right)}{\left(\left|z_{0}\right||\lambda|^{n_{k}}\right)^{\rho}}=\frac{L_{1}\left(z_{0}\right)}{\left|z_{0}\right|^{\rho}}=L\left(z_{0}\right) .
$$




\section{Conditions for the unboundedness of SOlutions AlOng RAYS}

Note that Theorem 3.1 and Corollary 4.1 are results of conditional type. In general, we do not know whether condition (3.4) is satisfied in a specific point $z_{0}=r_{0} e^{i \vartheta_{0}}$. In this section we give some conditions under which any non-trivial entire solution $f(z)$ of $(3.1)$ is unbounded on any ray $\arg z=\vartheta$. Denote as before $\lambda=|\lambda| e^{2 \pi i \beta}$.

We can assure the unboundedness of $f(z)$ along all rays under the following conditions either on $|\lambda|$ or on $\arg \lambda$ :

a) $|\lambda|$ is large compared to $m=\operatorname{deg} P(z)$

b) $\beta$ is rational, $\beta=t / s$ (in lowest terms) and $s$ is large compared to $\rho=\frac{\log m}{\log |\lambda|}$

c) $\beta$ is irrational.

The proofs of b) and c) are based on the Phragmén-Lindelöf principle, or more precisely on the following corollary.

Corollary 5.1 (Phragmén-Lindelöf, [10]). Assume that $f(z)$ is a non-constant entire function of order $\rho \geq \frac{1}{2}$. Suppose that $n$ rays emanate from the origin and split the complex plane $\mathbb{C}$ into $n$ angular regions each of which has angle less than $\frac{\pi}{\rho}$. Then $f(z)$ is unbounded on at least one of these rays.

\subsection{Large $|\lambda|$.}

Theorem 5.1. Suppose that $|\lambda|>m^{2}$, i. e. $\rho<\frac{1}{2}$. Then

(1) $f(z)$ is unbounded along any ray $\arg z=\vartheta$

(2) For any $\vartheta$ there exists a sequence $r_{n}=r_{n}(\vartheta) \rightarrow \infty$ such that the limit

$$
\lim _{n \rightarrow \infty} \frac{\varphi\left(r_{n} e^{i \vartheta}\right)}{r_{n}^{\rho}}=L(\vartheta)
$$

exists.

(3) These limits $L(\vartheta)$ are bounded between two positive constants for all $\vartheta \in[0,2 \pi]$.

(4) Under the additional assumption that $\beta=\frac{1}{2 \pi} \arg \lambda$ is rational $\left(\beta=\frac{t}{s}\right.$ in lowest terms) further refinements are possible. Denote $q=\lambda^{s}$ (and note that $q>1$ ). Then $r_{n}$ can be chosen as a geometric progression $r_{n}=r_{0} q^{n}$ independent of the direction $\vartheta$.

Proof. Unboundedness of $f(z)$ along any ray follows from the assumption $\rho<\frac{1}{2}$ (see [14, 8.73]). Moreover there exists a sequence $\tilde{r}_{n} \rightarrow \infty$ such that

$$
m\left(\tilde{r}_{n}\right)=\min _{|z|=\tilde{r}_{n}}|f(z)| \rightarrow \infty
$$

In particular, one can find a circle $C_{0}=\left\{z \in \mathbb{C}|| z \mid=r_{0}\right\}$ such that condition (3.4) is satisfied for all $z \in C_{0}$. Then (2) and (3) follow either from Corollary 4.1 (if $\beta$ is rational) or from Theorem 4.3 (if $\beta$ is irrational). Applying Corollary 4.1 once again yields (4). 


\subsection{Rational $\beta$ with large denominator.}

Theorem 5.2. Suppose that $\beta=\frac{1}{2 \pi} \arg \lambda$ is rational ( $\beta=\frac{t}{s}$ in lowest terms). Suppose further that $s>2 \rho$. Then

(1) $f(z)$ is unbounded on any ray $\arg z=\vartheta$.

(2) Furthermore, for any $\vartheta$ one can find a geometric progression $r_{n}=r_{n}(\vartheta)=q^{n} r_{0}(\vartheta)$ (with $q=\lambda^{s}>1$ ) such that the limit (5.1) exists and $L(\vartheta)>0$.

Proof. Suppose that there exists a ray $\arg z=\vartheta_{1}(r>0)$ such that $f(z)$ is bounded along this ray. Then in view of equation (3.1) $f(z)$ is bounded along all rays $\arg z=\vartheta_{1}+2 k \pi \frac{t}{s}$ $\bmod 2 \pi(k=0, \ldots, s-1)$. The angle between two consecutive rays is $\frac{2 \pi}{s}<\frac{\pi}{\rho}$. From Corollary 5.1 it follows that $f$ is constant. Assertion (2) follows by applying Corollary 4.1 as in the proof of Theorem 5.1.

5.3. Irrational $\beta$. Suppose that $\beta=\frac{1}{2 \pi} \arg \lambda$ is irrational. It was already proved in Section 4.2 as an immediate consequence of Theorem 4.3 that every non-trivial entire solution $f(z)$ of (3.1) is unbounded along any ray $\arg z=\vartheta$. Here we present an alternative proof of this statement using again the Phragmén-Lindelöf principle. However the existence of the limit (4.9) cannot be obtained in this way.

Theorem 5.3. If $\beta$ is irrational, then every non-trivial entire solution $f(z)$ of $(3.1)$ is unbounded along any ray $\arg z=\vartheta$.

Proof. Suppose that $f(z)$ is bounded on a ray $\arg z=\vartheta_{1}$. Then again by (3.1) $f(z)$ is bounded along all rays $\arg z=\vartheta_{1}+2 k \pi \beta \bmod 2 \pi$ for $k \in \mathbb{N}$. Since the angles $\vartheta_{1}+2 k \pi \beta$ mod $2 \pi$ are dense in $[0,2 \pi]$, one can find $n$ such rays with angles between consecutive rays less than $\frac{\pi}{\rho}$. Corollary 5.1 implies then that $f(z)$ is constant.

\section{ReAl CASE: REAL $\lambda>1$ AND POlynomial $P(z)$ With REAL COEFFiCiENTS}

Throughout this section we will assume that $\lambda>1$ is real and all coefficients of $P(z)$ are real. Also we use the notations of Section 2 above.

Immediately, from Lemma 2.1 (points (3) and (4)) we derive

Corollary 6.1. Suppose that

(1) $p_{i} \geq 0$ for $i \geq 2$

(2) $f_{0} \geq 0$ and $f_{n_{0}}>0$.

Then

a) $f_{i} \geq 0$ for all $i$

b) there are infinitely many $i$ for which $f_{i}>0$

c) $f(z)>0$ for all $z \in \mathbb{R}^{+}$.

From this we obtain

Corollary 6.2. Under the assumptions of Corollary $6.1 f(z) \rightarrow \infty$ in $\mathbb{R}^{+}$. Moreover,

$$
f(r)=M(r)=\max _{|z|=r}|f(z)|
$$


and

$$
\log f(r) \sim r^{\rho} Q\left(\frac{\log r}{\log \lambda}\right) \text { for } r \rightarrow \infty,
$$

where $Q$ is a continuous 1-periodic function bounded between two positive constants.

Further refinements are possible under additional assumptions (cf. also [3, Theorem 1]).

Theorem 6.1. Assume that

(1) $\lambda>m$

(2) $p_{i} \geq 0$ for $i \geq 2$

(3) all preimages of 0 under $P$ are real.

Let $f(z)$ be an entire solution of (3.1) such that $f(0)$ and $f^{\prime}(0)$ are real and $f(0) \geq 0$ and $f^{\prime}(0)>0$. Then $f(z)$ tends to infinity along any ray in the sector $|\arg z|<\frac{\pi}{2}$. Moreover,

$$
\log \left|f\left(r e^{i \vartheta}\right)\right| \sim r^{\rho} Q_{\vartheta}\left(\frac{\log r}{\log \lambda}\right) \text { for } r \rightarrow \infty,
$$

where $Q_{\vartheta}$ is a 1-periodic function bounded between two positive constants.

The proof is based on the following three lemmas.

Lemma 6.1. Suppose that all preimages of 0 under $P$ are real. Let $f(z)$ be an entire solution of (3.1) such that $f(0)$ and $f^{\prime}(0) \neq 0$ are real. Then $f(z)$ has only real zeros.

Proof. Suppose that $f(z)=0$ has a non-real solution $z_{0}$. Then

$$
0=f\left(z_{0}\right)=P\left(f\left(z_{0} \lambda^{-1}\right)\right)=\cdots=P^{(n)}\left(f\left(z_{0} \lambda^{-n}\right)\right),
$$

which implies that $f\left(z_{0} \lambda^{-n}\right)$ is an $n$-th preimage of 0 under $P$ and is therefore real by our assumptions. Using Taylors formula we obtain

$$
\lim _{n \rightarrow \infty} \lambda^{n}\left(f\left(z \lambda^{-n}\right)-f(0)\right)=f^{\prime}(0) z .
$$

Here the left hand side is real and the right hand side is not, which gives a contradiction.

Lemma 6.2. Suppose that the assumptions of Lemma 6.1 are satisfied. Assume further that

(1) $p_{i} \geq 0$ for $i \geq 2$

(2) $f(0) \geq 0$ and $f^{\prime}(0)>0$.

Then $f(z)$ has non-positive real zeros only.

Proof. The assumption $f^{\prime}(0) \neq 0$ implies that $n_{0}=1$. Furthermore, we have $f_{n_{0}}>0$. Thus by Corollary $6.1 f(z)>0$ on $\mathbb{R}^{+}$. Thus the zeros have to be non-positive.

Lemma 6.3. Assume that $f(z)$ is an entire function of order $0<\rho<1$ with only negative real zeros. Then $f(z)$ tends to infinity faster than any power of $z$ along any ray in the sector $|\arg z|<\frac{\pi}{2}$. 
Proof. Because $0<\rho<1$ according to Hadamard's theorem we have

$$
f(z)=\prod_{n=1}^{\infty}\left(1+\frac{z}{r_{n}}\right)
$$

where $\left(-r_{n}\right)_{n \in \mathbb{N}}$ with $r_{n}>0$ are the negative zeros of $f$. Write $z=x+i y$ and $S_{\vartheta}=\{z \in$ $\mathbb{C}|| \arg z \mid \leq \vartheta\}$ for $\vartheta<\frac{\pi}{2}$. Then we have for $z \in S_{\vartheta}$

$$
\left|1+\frac{z}{r_{n}}\right|>1+\frac{x}{r_{n}} \geq 1+\frac{|z| \cos \vartheta}{r_{n}} \geq \max \left(1, \frac{|z| \cos \vartheta}{r_{n}}\right)
$$

Inserting this estimate into (6.3) yields

$$
|f(z)|=\prod_{n=1}^{\infty}\left|1+\frac{z}{r_{n}}\right|>\prod_{n=1}^{N}\left|1+\frac{z}{r_{n}}\right|>C|z|^{N},
$$

where $C=\cos ^{N} \vartheta /\left(r_{1} \cdots r_{N}\right)$ and $N$ is an arbitrary positive integer.

Proof of Theorem 6.1. Combining Lemma 6.3 and Corollary 4.2 proves the theorem.

To conclude this section we present a lemma, which gives a condition for the reality of the preimages of 0 in the case of quadratic polynomials.

Lemma 6.4. Let

$$
P(z)=a z(z-\omega), \quad 0 \neq \omega \in \mathbb{R}
$$

All preimages of 0 under $P$ are real, if and only if the following condition is fulfilled

$$
a|\omega| \geq \begin{cases}2 & \text { for } \omega>0 \\ 4 & \text { for } \omega<0\end{cases}
$$

Proof. We will give a short geometric proof of this lemma using the plots of the functions $a z(z-\omega)$ for $\omega>0$ and $\omega<0$ in Figure 1.

We will give the proof for $\omega>0$; the proof for $\omega<0$ is similar.

The fixed points of $P(z)$ are 0 and $b=\omega+\frac{1}{a}$. Next, $P^{-1}(\{0\})=\{0, \omega\}, P^{-1}(\{b\})=$ $\left\{-\frac{1}{a}, b\right\}$, and $P^{-1}(\omega)$ consists of two points from the interval $I=\left[-\frac{1}{a}, b\right]$.

Now, to prove sufficience of (6.5) it is enough to prove that under condition (6.5) every preimage of a point of $I$ lies in $I$. As can be seen from Figure 1, this is true, if

$$
\frac{1}{a} \leq-\min _{z \in \mathbb{R}} P(z)=-P\left(\frac{\omega}{2}\right)=\frac{a}{4} \omega^{2},
$$

which is satisfied if $a \omega \geq 2$ and $\omega>0$. This proves sufficience of (6.5).

On the other hand, suppose that $a \omega<2$. Then

$$
\frac{1}{a}>-\min _{z \in \mathbb{R}} P(z)
$$

Then there exist points $c \in P^{-n}(\{0\})$ arbitrarily close to the fixed point $b$. Thus there exists a point $d \in P^{-1}(\{c\}) \subset P^{-n-1}(\{0\})$ arbitrarily close to $-\frac{1}{a} \in P^{-1}(\{b\})$. By (6.6) the preimages of $-\frac{1}{a}$ and therefore of $d$ are not real. Thus $P^{-n-1}(\{0\})$ contains non real points. This proves necessity of (6.5). 

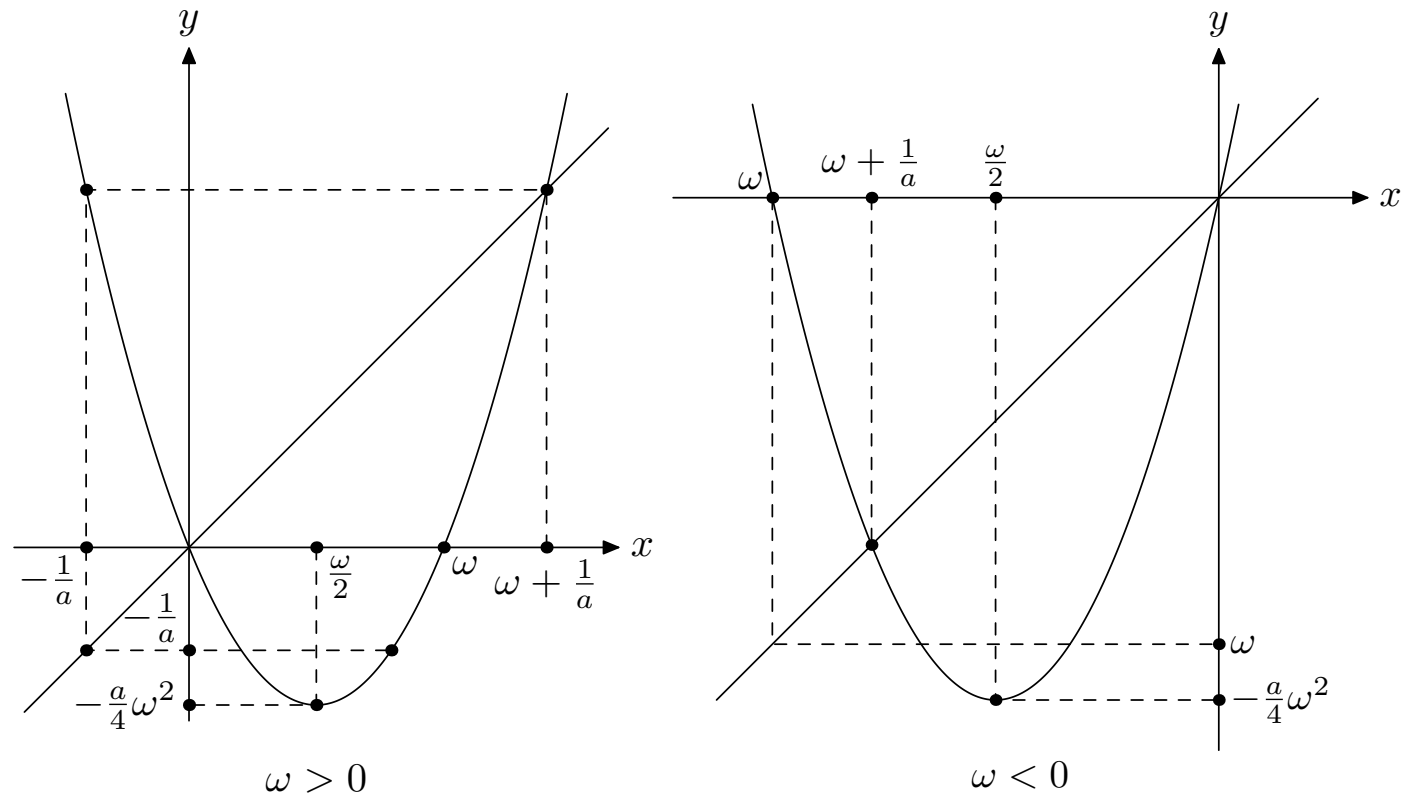

FiguRE 1. The graphs of $a z(z-\omega)$ for $\omega>0$ and $\omega<0$.

\section{Asymptotic Behaviour in Angular Regions}

In this section, as well as in Section 6 , we assume that $\lambda>1$ is real and all coefficients of $P(z)=z^{m}+p_{m-1} z^{m-1}+\cdots+p_{0}$ (see (3.2)) are real. Moreover, (by virtue of Proposition 2.3 and Remark 2.2) without loss of generality, we assume that $P(0)=0, P^{\prime}(0)=\lambda, f(0)=0$, and $f^{\prime}(0)>0$.

Let $f(z)$ be an entire solution of (2.1). In contrast with the previous sections (where asymptotics of $|f(z)|$ is studied) here we collect some results on the asymptotics of the solution $f(z)$ itself in some angular regions of the complex plane.

As a starting point we present a theorem, which we proved in [3].

Theorem 7.1 ([3, Theorem 1]). Let $f$ be an entire solution of the functional equation (2.1). Furthermore, suppose that $\mathcal{F}_{\infty}$, the Fatou component of $\infty$ of $P$, contains an angular region of the form

$$
W_{\beta}=\{z \in \mathbb{C} \backslash\{0\}|| \arg z \mid<\beta\}
$$

for some $\beta>0$. Then for any $\varepsilon>0$ and any $M>0$ the asymptotic relation

$$
f(z)=\exp \left(z^{\rho} Q\left(\frac{\log z}{\log \lambda}\right)+o\left(|z|^{-M}\right)\right)
$$

holds uniformly for $z \in W_{\beta-\varepsilon}$, where $Q$ is a periodic holomorphic function of period 1 on the strip $\left\{w \in \mathbb{C}|| \Im w \mid<\frac{\beta}{\log \lambda}\right\}$. The real part of $z^{\rho} Q\left(\frac{\log z}{\log \lambda}\right)$ is bounded between two positive constants; $Q$ takes real values on the real axis. 
Remark 7.1. Notice that the condition on the Fatou component $\mathcal{F}_{\infty}$ is used in the proof of this theorem to ensure that $f(z)$ tends to infinity in the angular region $W_{\beta}$. Therefore, this condition could be replaced by

$$
\lim _{z \rightarrow \infty} f(z)=\infty \text { for }|\arg z|<\beta .
$$

Remark 7.2. E. Romanenko and A. Sharkovsky have studied equation (2.1) on $\mathbb{R}$ (rather than on $\mathbb{C}$ ). Applying Sharkovsky's method of "first integrals" ("invariant graphs") they obtained not only an asymptotic formula of type (7.1) but a full asymptotic expansion for all solutions $f(x)$, such that $f(x) \rightarrow \infty$ for $x \rightarrow \infty$.

We will present a number of consequences of this theorem.

From Theorem 7.1 and Theorem 4.3 we obtain

Corollary 7.1. Let $f$ be a solution of (2.1). Assume that there exists $r>0$ such that

$$
\forall x \in[r, \lambda r]:|f(x)|>\max (e, 2 m K) .
$$

Then there exists $\gamma>0$ such that for any $0<\varepsilon<\gamma$ and any $M>0$ the asymptotic relation (7.1) holds uniformly for $z \in W_{\gamma-\varepsilon}$.

As an immediate corollary of Theorem 7.1 we get

Corollary 7.2. Let $f$ be a solution of (2.1). If the Julia set of $P$ is a subset of $\mathbb{R}^{-} \cup\{0\}$, then for any $\varepsilon>0$ and any $M>0$ the asymptotic relation (7.1) holds uniformly for $z \in W_{\pi-\varepsilon}$.

Acknowledgment. We are grateful to E. Romanenko and A. Sharkovsky for having sent their preprint and for stimulating discussions. We thank M. Sodin for useful remarks.

\section{REFERENCES}

1. M. T. Barlow and E. A. Perkins, Brownian motion on the Sierpiński gasket, Probab. Theory Relat. Fields 79 (1988), 543-623.

2. J. D. Biggins and N. H. Bingham, Near-constancy phenomena in branching processes, Math. Proc. Camb. Philos. Soc. 110 (1991), 545-558.

3. G. Derfel, P. J. Grabner, and F. Vogl, The zeta function of the Laplacian on certain fractals, Trans. Amer. Math. Soc ? (2006), ?-?, to appear.

4. G. Derfel and F. Vogl, Divide-and-conquer recurrences-classification of asymptotics, Aequationes Math. 60 (2000), 243-257.

5. A. Eremenko and G. Levin, Periodic points of polynomials, Ukrainian Math. J. 41 (1989), 1258-1262.

6. A. Eremenko and A. Sodin, Iterations of rational functions and the distributions of the values of poincare functions, J. Soviet Math. 58 (1992), 504-509.

7. P. J. Grabner, Functional iterations and Brownian motion on the Sierpiński gasket, Mathematika 44 (1997), 374-400.

8. P. J. Grabner and W. Woess, Functional iterations and periodic oscillations for the simple random walk on the Sierpiński graph, Stochastic Processes Appl. 69 (1997), 127-138.

9. S. Karlin and J. McGregor, Embeddability of discrete time simple branching processes into continuous time branching processes, Trans. Amer. Math. Soc. 132 (1968), 115-136.

10. A. I. Markushevich, Theory of functions of a complex variable. Vol. I, II, III, english ed., Chelsea Publishing Co., New York, 1977, Translated and edited by Richard A. Silverman. 
11. H. Poincaré, Sur une classe étendue de transcendantes uniformes, C. R. Acad. Sci. Paris 103 (1886), 862-864.

12. 316-365.

13. E. Romanenko and A. Sharkovsky, Long time properties of solutions of simplest q-difference equations (Russian), Preprint, 2000.

14. E. C. Titchmarsh, The theory of functions, 2nd corr. ed., Oxford University Press, 1979.

15. G. Valiron, Lectures on the General Theory of Integral Functions, Private, Toulouse, 1923.

16. G. Valiron, Fonctions analytiques, Presses Universitaires de France, Paris, 1954.

(G. D.) Department of Mathematics and Computer Science, Ben Gurion University of THE Negev, BeER Sheva 84105, IsRAel

E-mail address: derfel@math.bgu.ac.il

(P. G.) Institut für Mathematik A, Technische Universität Graz, Steyrergasse 30,8010 Graz, Austria

E-mail address: peter.grabner@tugraz.at

(F. V.) Institut für Analysis und Scientific Computing, Technische Universität Wien, Wiedner Hauptstrasse 8-10, 1040 Wien, Austria

E-mail address: fvogl@osiris.tuwien.ac.at 